\title{
STUDY OF QUASI-FRACTAL MANY-PARTICLE-SYSTEMS AND PERCOLATION NETWORKS BY ZERO-LOSS SPECTROSCOPIC IMAGING, ELECTRON ENERGY-LOSS SPECTROSCOPY AND DIGITAL IMAGE ANALYSIS
}

\author{
Vladimir P. Oleshko ${ }^{1 * * *}$, Volodymyr V. Kindratenko ${ }^{2}$, Renaat H. Gijbels ${ }^{2}$, \\ Pierre J.M. Van Espen ${ }^{2}$, and Wim A. Jacob ${ }^{3}$ \\ ${ }^{1}$ N.N. Semenov Institute of Chemical Physics, Russian Academy of Sciences, \\ 117421 Moscow, Russia. \\ ${ }^{2}$ Department of Chemistry and ${ }^{3}$ Department of Medicine, University of Antwerp (U.I.A.), \\ B-2610 Wilrijk-Antwerpen, Belgium.
}

\begin{abstract}
Submicron colloidal Ag particles and nano-sized filaments forming a statistical percolation network during "in situ" development of double structure tabular microcrystals of $\mathrm{AgBr}(\mathrm{I})$ emulsions have been studied by electron energy-loss spectroscopy and zero-loss electron spectroscopic imaging (EELS/ ZLESI). Image analysis has shown that random quasi-fractal clusters were formed in the colloid. ZLESI has been applied to characterise the morphology and defect structure of aggregated particles and filaments. Their energy-loss spectra revealed plasmon excitations and interband $4 \mathrm{~d}$ electron transitions between 4-32 eV energy-loss. To study the cluster structure and its relation to the physical properties, fractal analysis including estimations of cluster fractal dimensions and of density autocorrelation functions has been performed. Mechanisms of fractal aggregation based on known models of diffusion limited aggregation, cluster-cluster aggregation and percolation are discussed.
\end{abstract}

Key words: small particles, EELS/ZLESI, image analysis, fractal clusters, percolation networks.

\section{$\underline{\underline{\text { Introduction }}}$}

Nowadays coagulated fractal and quasi-fractal structures have been an area of active research [1-3]. They can result from a variety of different processes: film deposition on a solid surface, adsorption of molecules by porous matter, solidification of colloids, crystallization of ultradisperse powders, various biophysical processes, etc. As a rule, fractal properties at objects having porous, ramified, rough or sparse and tenuous structures are usually associated with nonequilibrium growth [4]. The unique physical and chemical properties, different from those of both gases and condensed media make fractal clusters of great fundamental interest. Moreover, fractal properties can be used for the characterisation of many-particle-systems and microanalysis of surfaces and various disperse substances as well. Volume and surface plasmon excitations, in particular, are determined both by properties of the individual particles and by collective effects due to interaction among the particles densely packed in aggregates. To understand how fractal geometry affects electronic properties of aggregated matter, electron-optical and optical methods can be applied [3,5].

Here we present some data on formation of quasi-fractal clusters in aggregated Ag colloids and of percolation filament networks during development of composite tabular microcrystals of $\mathrm{Ag}(\mathrm{Br}, \mathrm{I})$ emulsions studied by electron energy-loss spectroscopy (EELS), zero-loss electron spectroscopic imaging (ZLESI) and image analysis.

\section{Experimental}

A suspension of colloidal $\mathrm{Ag}$ particles was obtained by reduction of a diluted aqueous solution of $\mathrm{AgNO}_{3}$ in the presence of $0.1 \%$ polyvinyl alcohol (PVA). In another experiments statistical networks

\footnotetext{
*Present address: $\quad$ Department of Chemistry, University of Antwerp (U.I.A.), Universiteitsplein 1, B-2610 Antwerpen-Wilrijk, Belgium.

${ }^{* *}$ To whom correspondence should be addressed.
} 
of Ag filaments were produced by a 2-minute "in situ" treatment, with the Kodak D-19 developer, of double structure tabular microcrystals; the latter had a size of 7-10 $\mu \mathrm{m}$ and consisted of $\mathrm{AgBr}$ cores and $\operatorname{Ag}(\mathrm{Br}, \mathrm{I})$ shells containing 5-6 mol.\% of AgI. Samples for observations were prepared by deposition of particles and developed crystals on object grids covered with thin carbon films. A ZEISS EM 902 electron microscope with an integrated prism-mirror-prism energy filter operated at $80 \mathrm{kV}$ accelerating voltage was used for EELS/ZLESI measurements. EELS spectra were acquired using a photomultiplier serial detector with a collection half-angle of $11 \mathrm{mrad}$. The exit energy-selecting slit in the image mode was $10 \mathrm{eV}$. The analyses were performed with an energy resolution of $1.5-2.5 \mathrm{eV}$ on the full-width at half-maximum of the zero-loss peak. EDX spot analysis of the specimens was performed in the STEM mode at $80 \mathrm{kV}$ accelerating voltage using a JEOL 1200EX electron microscope equipped with an EM-ASID10 scanning observation device and a TRACOR NORTHERN TN5500 energy dispersive X-ray analyzer.

Digital images of particles were acquired via a SIT TV camera by a KONTRON IBAS 2000 image analysis system. Quantitative image processing was done to perform fractal analysis of particle aggregates as well as to estimate the parameters of the size distribution of the particles. Selected fields in the latter case contained 500 distinguishable particles.

In order to separate particles from their background, a technique described in [6] has been applied. The method is based on the idea of a similarity of the original image and obtained from its binary ones. The similarity is measured in terms of the correlation, so from all possible binary images obtained from the original one using different global threshold values only the image having highest correlation between them and the original one is used as the binary image. The sensitivity of the results to the accuracy of the background subtraction technique was tested and described in an earlier publication [7]. A border following algorithm [8] has been used for the contour extraction. Estimation of fractal dimensions, $\boldsymbol{D}$, has been performed using the following techniques: (1) the "hybrid" method searching the relation, $\boldsymbol{P} \sim \boldsymbol{S}^{\boldsymbol{D}-1}$, between the perimeter of the cluster's projection on an image plane, $\boldsymbol{P}$, and a "yardstick" size, $\boldsymbol{S}$, used for the measurement of the perimeter [9]; (2) the box counting method based on the relation, $\boldsymbol{N} \sim \boldsymbol{S}^{-D}$, between the number of boxes, $\boldsymbol{N}$, covering the cluster projection contour on the image plane and their side, $\boldsymbol{S}$ [10]; (3) the covering set method using the relation, $\boldsymbol{N} \sim \boldsymbol{S}^{-\boldsymbol{D}}$, between the number of occupied sites, $\boldsymbol{N}$, covering the cluster projection and their side, $\boldsymbol{S}[10]$; (4) the Forrest-Witten technique [11] based on the relation, $\boldsymbol{N} \sim \boldsymbol{S}^{\boldsymbol{D}}$, between the number of primary particles, $N$, within a square of side, $S$, plotted on the projection plane of the cluster. The density autocorrelation analysis was done to evaluate the relation, $\boldsymbol{C}(\boldsymbol{r}) \sim \boldsymbol{r}^{\boldsymbol{D}-d}$, between the average density, $\boldsymbol{C}(\boldsymbol{r})=0.5 p r N S<p\left(r+r^{\prime}\right) p\left(r^{\prime}\right)>$, of occupied points in the projection plane of the cluster at a distance $r$. Here $\boldsymbol{N}$ is the total number of occupied sites, $\boldsymbol{p}\left(\boldsymbol{r}^{\prime}\right)$ is the density at a reference point vector $\boldsymbol{r}^{\prime} ; \boldsymbol{p}\left(\boldsymbol{r}+\boldsymbol{r}^{\prime}\right)$ is the density at vector $\boldsymbol{r}+\boldsymbol{r}^{\prime}$ equal 1 (occupied point) or 0 (free point); $\boldsymbol{d}$ is dimension of space $(\boldsymbol{d}=2)$. Testing of some techniques on a Sierpinsky carpet with the fractal dimension 1.89 gave the next results: $1.80 \pm 0.19$ (the covering set method); $1.89 \pm 0.13$ (the Forrest-Witten technique); $1.91 \pm 0.11$ (the pair correlation function analysis). Analysis of the triadic Koch curve with theoretical fractal dimension 1.26 gives the following results: 1) $1.25 \pm 0.07$ (the "hybrid" method); 2) $1.26 \pm 0.15$ (the box counting method).

\section{$\underline{\underline{\text { Results and Discussions }}}$}

\section{$\underline{\text { Ag Colloids }}$}

TEM micrographs of colloidal Ag particles are shown in Fig. 1. A zero-loss filtered image (b) demonstrated a higher contrast and better resolution than a corresponding unfiltered one (a) because the blurring and the chromatic aberration caused by inelastically scattered electrons in the specimen is avoided. This allowed to observe more clearly grain boundaries and crystalline defects, e.g. twins and stacking faults. Fig. 1c shows a low magnification view of a cluster formed by colloidal particles. The inset presents a discrete ring zero-loss spectroscopic selected-area electron diffraction pattern of 
aggregated particles pointing to a face centered cubic polycrystalline structure. Zero-loss filtering in this case removed the inelastically scattered electrons, which contribute to the background, and increased the contrast of the diffraction patterns. The particle size distribution could satisfactorily be fitted to a log-normal curve with a mean size of $42.7 \pm 2.2 \mathrm{~nm}$.

Usually the particles in such colloids are prevented from aggregation by repulsive forces between double layers at the particle surfaces. These forces overcome the Van der Waals attraction. Addition of ions to the colloidal system affects the space charge of the particle surfaces and induces aggregation. Initially this leads to coagulation aggregates. Neighbouring particles in the aggregates are separated by thin ion shells at their surface. By aging of the colloidal system the neighbouring particles coalesce partially and build a common grain boundary. The pictures in Fig.1 demonstrate the topological arrangement of a statistical network consisting of random aggregates and irregular chains of coagulated particles. The aggregate shown in Fig. 1c apparently consists of three parts formed at earlier stages by cluster-particle aggregation. The actual aggregation cannot adequately be described by a model of cluster-particle (CPA) or cluster-cluster (CCA) aggregation only. Real processes may involve these two mechanisms occurring simultaneously or sequentially and/or more complicated aggregation mechanisms. The average density of the aggregate, estimated by the autocorrelation density technique, decreased with increasing radius of gyration in accordance with the CCA model which preferentially contributes to the final stage.

An essential feature of the studied colloids is a power law relationship $\boldsymbol{M} \sim \boldsymbol{R}^{D \boldsymbol{\beta}}$ between the mass, $\boldsymbol{M}$, (or the number of particles) and the radius of gyration, $\boldsymbol{R}$, of the aggregate (see Fig. 2a). Here $\boldsymbol{D}_{\boldsymbol{\beta}}$ is the mass fractal dimension characterising a given population of aggregates. It is significant that at the early stages of aggregation clusters forming by the CPA mechanism contain a small number (100300) of specks and therefore cannot be considered as fractals in the strict sense of the word [12]. In this case the parameter $\boldsymbol{D}_{\boldsymbol{\beta}}$ describes the distribution of the substance in the system. However, the high values of the correlation coefficient of linear regression (0.98) indicate that even in this range the fractal approach is most appropriate for describing disordered aggregated structures. Analysis of a population of 39 clusters gave the value $\boldsymbol{D}_{\boldsymbol{\beta}}=1.81 \pm 0.11$. This result can be compared with the value $1.81 \pm 0.05$ [13] obtained for computer simulated diffusion limited aggregation (DLA) growth of fractal clusters along random linear trajectories. The model developed for describing the 3D cluster aggregation was shown to be in agreement with results obtained by the Forrest-Witten technique [11]. Since we consider preferentially 2D aggregated structures, the cluster mass determined from the projection will be approximately equivalent to the total mass of the object in three dimensions. Also, the radius of gyration, obtained from the projection, will be essentially the same value determined from the 3D structure. Thus the projection demonstrates the same behaviour as the corresponding 3D cluster. This may explain why our data are in good agreement with results for the simulated 3D cluster aggregation. The values of the fractal dimension obtained by the Forest-Witten technique, which characterise the internal automodeling of separate aggregates (known as the box fractal dimension, $\boldsymbol{D}_{\boldsymbol{\alpha}}$, ( $\boldsymbol{D}_{\boldsymbol{\alpha}}=\boldsymbol{D}_{\boldsymbol{\beta}}$ for self-similar fractals), were in the range 1.86-1.91. Some differences between $\boldsymbol{D}_{\boldsymbol{\beta}}$ and $\boldsymbol{D}_{\boldsymbol{\alpha}}$ were referred to a disturbance of the inner cluster automodeling.

Most of quasi-fractal clusters analyzed by the "hybrid" method exhibited contour fractal dimension values in the range from 1.20 to 1.35 . The values obtained by the box counting methods were 1.30-1.45. This reflects an irregular shape of the outer border cluster structures. The interpretation of these data, which, in principle, can be used for the classification of the structures studied, requires further analysis including also an evaluation of boundary effects.

Fig. 2b, curve 1 presents a single scattering distribution derived from an initial EELS spectrum of a quasi-fractal aggregate by Fourier-log deconvolution [14]. Unfortunately, the spectrum was governed in the low-loss region by the carbon plasmon contribution from the support. A shift of the plasmon peak at $21.5 \mathrm{eV}$ to lower energy-losses compared with the value $25 \mathrm{eV}$ for pure carbon film probably was due to the contribution of surface plasmons excited in the aggregated particles and in the PVA layer [5]. 


\section{$\underline{\text { Ag Filament Networks }}$}

Fig. 3a shows a ZLESI image of the central part of a double structure tabular microcrystal after a 2minute "in situ" treatment with the Kodak developer D-19. Development of crystals was usually accompanied with an intensive etching and resulted in the formation of a network of Ag filaments in the core region [15]. Here again zero-loss filtering allowed to improve the contrast and resolution of filament fine features, i.e. grain boundaries, twins and stacking faults. The development process in the shell regions occurred more slowly and etching was not so intensive because of the lower solubility of $\mathrm{AgBr}_{1-\mathrm{x}} \mathrm{I}_{\mathrm{x}}$ as compared with pure $\mathrm{AgBr}$. In the central part of the crystal, Ag filaments and particles formed branched web-like statistical networks. Generation of disordered aggregated structures by silver filaments and particles is obviously related to the stochastic nature of the development process in specific conditions, i.e., the local excess of the active volume developer and the high overpotential of the anode step of the reaction, the occurrence of both direct chemical and solution physical development mechanisms, and the presence of a protective polymer layer on the crystal surfaces [16]. Moreover, the obtained results suggest that the networks arise in some type of critical process, where such features as self-similarity, scaling and universality are displayed, and which, in principle, may be suitably described by percolation [17]. The power-law behaviour of the silver mass, $N$, (the number of occupied sites) vs. the square size, $\boldsymbol{S}$, shown in Fig. 3 b points out that the networks belong to random fractals. The fractal dimension, $\boldsymbol{D}_{\boldsymbol{\alpha}}$, determined from the slope of the curve was $1.95 \pm 0.11$. Numerical computations of the network growth within a simplified invasion percolation model [18] shown in Fig. 3 describe, respectively, an initial stage (c) and a final stage (d) of the aggregation process with a hexagonal lattice. At the final stage a simulated extended network obviously exhibits the morphology of filaments similar to that on the ZLESI image in Fig. 3a. The value of $\boldsymbol{D}_{\boldsymbol{\alpha}}$ for the simulated network shown in Fig.3d was $1.94 \pm 0.11$ in satisfactory agreement with the experimental value (see Fig.3b). It was also found that in both cases the autocorrelation function, $\boldsymbol{C}(\boldsymbol{r})$, demonstrates a similar behaviour. Based on the obtained results, one can conclude that this model can be applied to describe the filament network growth in the course of the photographic development process.

Numerous chemically produced contact areas within numerous filaments allowed to reduce the total electrical resistivity of the system. Above the percolation threshold they formed extended conducting paths within the network of randomly connected filaments. The correlation length of such paths increased with increasing filling factor and could promote the direct electrical $d c$ conductivity [3]. On the other hand, it should be noted that the growth of the developed silver phase involves a series of complicated cooperative transformations of preferentially ionic bonding in silver halide to metal bonds within the networks of nano-sized silver filaments and particles. This leads to an overlapping of molecular orbitals of primary silver clusters and to the formation, in the growing filaments, of an extended band structure involving, in particular, a narrow $4 \mathrm{~d}$ band separated from the conduction band and a number of surface and defect states. Drastic changes of the electronic structure, obviously affected in this case by the high dispersity of the matter, could be characterised by EELS.

The enlarged low-loss part of the spectrum of filaments formed in the crystal core (the recorded area was $0.8 \mu \mathrm{m}$ in diameter) contained a series of energy-loss maxima between 4 and $32 \mathrm{eV}$ (see Fig.2b, curve 2). Removing the plural scattering contribution from the initial spectrum shows that these multiple losses are due to oscillation of the imaginary part of the energy-loss function $\operatorname{Im}[-$ $1 / \varepsilon(E)]$ caused by interband electron transitions [15]. In this case the energy of the transition from the $4 \mathrm{~d}$ band to the Fermi level $(\geq 3.9 \mathrm{eV})$ is not small compared to the plasma energy of the free electron gas with a density of one valence electron per atom of silver $(9 \mathrm{eV})$. As a result, the surface loss $(\varepsilon=-1$, $\mathrm{h} v=3.6 \mathrm{eV})$ and the volume loss $(\varepsilon=0, \mathrm{~h} v=3.8 \mathrm{eV})$ are close together. Therefore, the plasma resonance at about $4.0 \mathrm{eV}$ as well as the "free-electron" resonance at $7 \mathrm{eV}$ were detected. This was referred to collective excitations of underlying $4 \mathrm{~d}$ electron shells of low binding energy mixed with the conduction band which could be promoted by the extended electrical dc-percolation [3]. 
Energy-loss spectra of developed crystals revealed also weak bands between 50 and $80 \mathrm{eV}$, which were attributed to the minor delayed $\mathrm{Ag} \mathrm{N}_{2,3}$-edge at about $60 \mathrm{eV}$. This was confirmed by EDX spot analysis of the crystals, which showed an increased intensity of the Ag L-series (Ag L $\alpha$ at 2.98 $\mathrm{keV}$ ) and the presence of bromide (the $\mathrm{Br} L \alpha$ at $1.48 \mathrm{keV}$ ) and traces of iodide (the I L $\alpha$ at $3.94 \mathrm{keV}$ ) from silver halide residuals. In accordance with data of the EDX analysis, the weak I $\mathrm{N}_{4,5}$-edge at about $50 \mathrm{eV}$ and the $\mathrm{Br} \mathrm{M}_{4,5}$-edge at about $72 \mathrm{eV}$ [15] could also contribute in this spectral region.

\section{$\underline{\underline{\text { Conclusions }}}$}

This study shows that EELS/ZLESI and digital image analysis are useful for the characterisation of coagulated Ag colloids and of chemically generated statistical networks of Ag filaments formed during "in situ" development of composite $\mathrm{Ag}(\mathrm{Br}, \mathrm{I})$ tabular microcrystals. Data presented point out that aggregated Ag particles of $42.7 \pm 2.2 \mathrm{~nm}$ in size deposited onto an inert carbon support form random quasi-fractal clusters. The mass fractal dimension of the clusters was estimated as $1.81 \pm 0.11$ in line with data on simulation of the fractal cluster growth according to the DLA model.

Box fractal dimension of a random network of nano-sized Ag filaments formed in the course of development of double structure tabular microcrystals of $\mathrm{Ag}(\mathrm{Br}, \mathrm{I})$ emulsions was determined as $1.95 \pm 0.11$ in satisfactory agreement with numerical computations of the network growth within the invasion percolation model. Energy-loss spectra of the percolation networks revealed plasmon excitations and interband electron transitions in the range from 4 up to $32 \mathrm{eV}$ energy-loss. This was attributed to collective excitations of underlying $4 \mathrm{~d}$ shells of low binding energy which probably were promoted by the extended electrical dc-percolation.

\section{$\underline{\underline{\text { Acknowledgements }}}$}

One of authors (1) was supported by the Belgian Federal Services for Scientific, Technical and Cultural Affairs (DWTC/SSTC) of the Prime Minister's Office through IUAP-III, Convention-49 and by the Russian Foundation for Basic Researches, grant No 95-03-08252.

\section{$\underline{\underline{\text { References }}}$}

[1] J.-P.Chevalier, C.Colliex, M.Tence, J.Microsc. Spectrosc. Electron., 1985, 10, 417.

[2] M.Tence, J.-P.Chevalier, R.Julien, J.Physique, 1986, 47, 1989.

[3] D. Schonauer, M. Quinten, U. Kreibig, Z. Phys. D, 1989, 12, 527.

[4] L.M. Sandler, Nature, 1986, 322, 789.

[5] H. Seiler, U. Haas, B. Ocker, K.-H. Kortje, Faraday Discuss., 1991, 92, 121.

[6]A.D. Brink, Pattern Recognit. Lett., 1989, 9, 335.

[7] V.V. Kindratenko, P.J. Van Espen, B.A. Treiger, R.E. Van Grieken, Environ. Sci. Technol., 1994, 28, 2194.

[8] A. Rosenfeld, A.C. Kak, Digital Picture Processing, Academic Press, London, 1982, v. 2, p. 219.

[9] N.N. Clark, Powder Technol., 1986, 46, 45.

[10] T. Cleary, R. Samson, J.W. Gentry, Aerosol Sci. Technol., 1990, 12, 518.

[11] S.R. Forrest, T.A.Witten, J. Phys. A: Math. Gen., 1979, 12, L109.

[12] V.P. Oleshko, M.V. Alfimov, X.-F. Cui, S.-E.Wang, J. Phot. Sci., 1994, 42, 110.

[13] P. Meakin, J. Col. Interf. Sci., 1984, 102, 491, 505.

[14] R.F. Egerton, Z.L.Wang, Ultramicroscopy, 1990, 32, 137.

[15] V. Oleshko, R. Gijbels, W. Jacob, F. Lakiere, A. Van Daele, E. Silaev, L. Kaplun, Microsc. Microanalys. Microstruct., 1995, 6, 1.

[16] V.P. Oleshko, M.V. Alfimov, J. Imag. Sci.\&Techn., 1994, 38, 162.

[17] T. Nakayama, K. Yakudo, L. Orbach, Rev. Mod. Phys., 1994, 66, 381.

[18] D. Wilkinson, M. Barsony, J. Phys., 1984, A17, L129. 
Microchimica Acta, 1996, suppl. 13, pp. 444-451. 


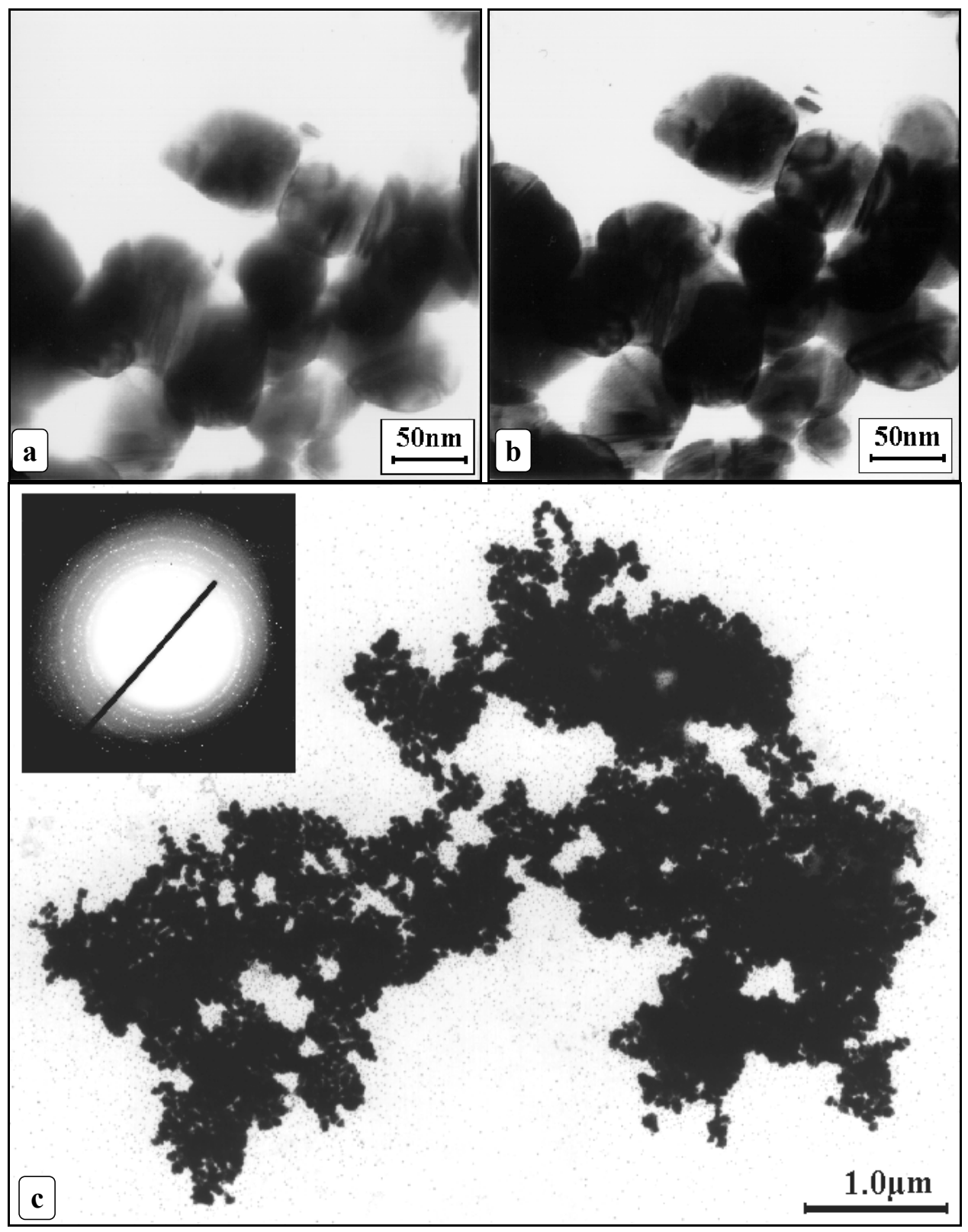

Figure 1. TEM images of colloidal Ag particles stabilized with a protective polymer: a) CTEM image; b) ZLESI image; c) ZLESI image of a fractal cluster formed by the particles. Left inset shows a zero-loss spectroscopic selected-area electron diffraction pattern. 


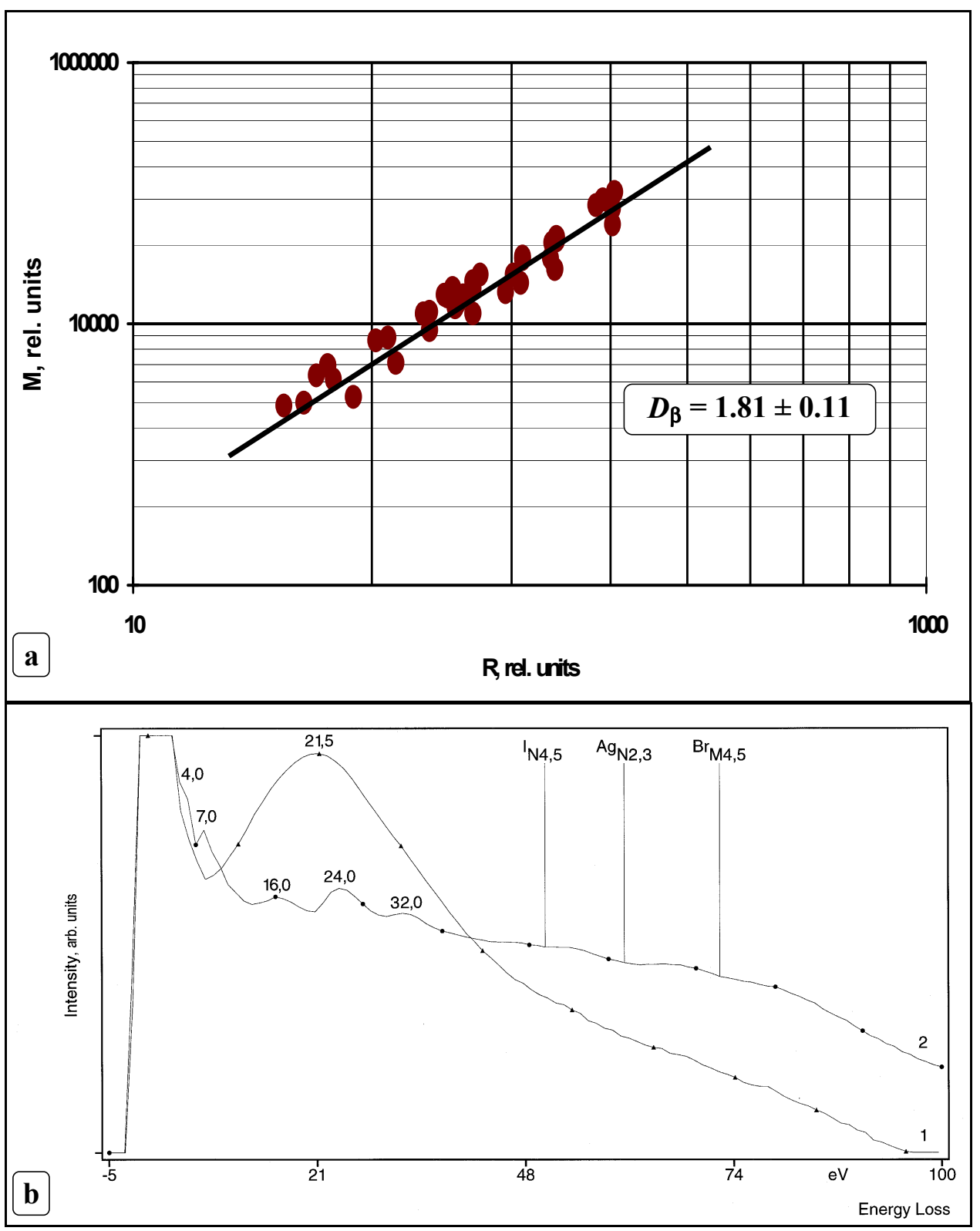

Figure 2. a) Mass dependence, $\boldsymbol{M}$, of the clusters vs. their size, $\boldsymbol{R}$; b) single scattering distributions derived from initial EELS spectra of colloidal Ag particles (curve 1) and filaments (curve 2). 

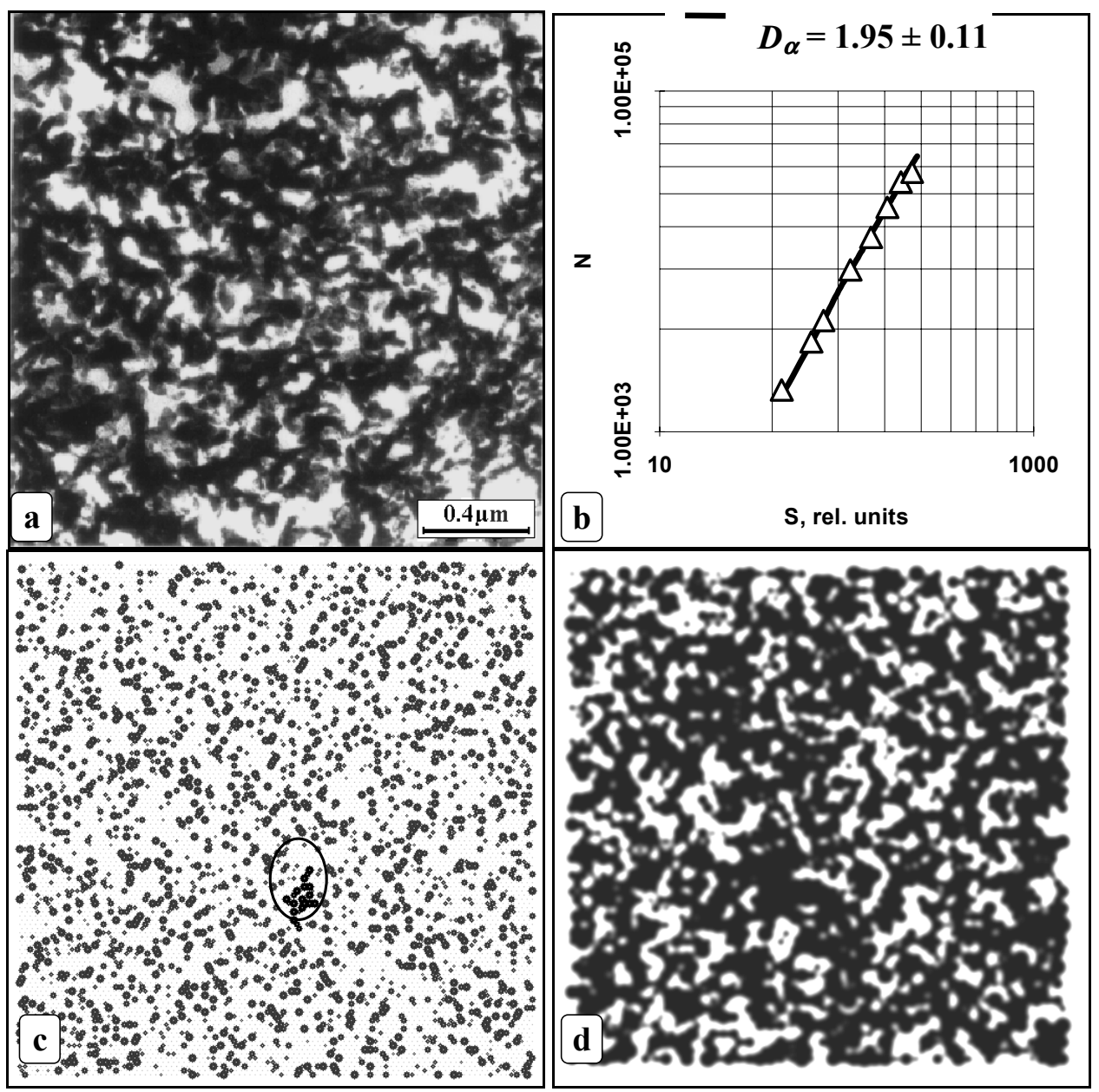

Figure 3. a) ZLESI micrograph of a network of Ag filaments formed in the course of a 2-minute development of a double structure $\mathrm{Ag} \mathrm{Br}(\mathrm{I})$ tabular microcrystal; (b) the number of occupied sites, $N$, vs. the square size, $S$, plotted on the image of the network; (c) computer simulation of growth of a percolation network (initial stage, a primary cluster is marked by the ellipse, probability of the occupation of a lattice site, $\boldsymbol{p}=0.2$ ); d) computer simulation (final stage, formation of the branched network, $\boldsymbol{p}=0.7$ ). The line in graph (b) corresponds to the experimental image (a), points obtained by the treatment of the simulated image (d) are shown by triangles. 